\title{
Novel nuclear materials characterization workflows enabled by fs-laser milling
}

Stephen Kelly ${ }^{1}$, Robin White ${ }^{1}$, Tobias Volkenandt $^{2}$, William Harris ${ }^{3}$, Benjamin Tordoff ${ }^{2}$, Giuliano Laudone $^{4}$, Katie Jones ${ }^{4}$ and Ben Veater ${ }^{4}$

${ }^{1}$ Carl Zeiss Microscopy GmbH, United States, ${ }^{2}$ Carl Zeiss Microscopy GmbH, Germany, ${ }^{3}$ Carl Zeiss Research Microscopy Solutions, United States, ${ }^{4}$ University of Plymouth, United States

Research to support nuclear energy development faces many challenges. Understanding material microstructures is not only essential to predicting and understanding the in-service performance of materials used in nuclear energy production, but also in understanding aging and corrosion of these materials as they interact with their environment. However, microstructural characterization of nuclear materials poses unique obstacles. Unique materials and material combinations push traditional microstructural evaluation techniques to their limits. Radioactive samples make normally routine microstructural characterization tasks much more complex. Precious samples force rigorous, multi-scale analysis workflows. And, materials that face and must endure uniquely harsh operational environments increase the demands for deep microstructural understandings. In this context, multiscale characterization workflows and the technology that supports them play an integral role in advancing materials development for nuclear energy production.

The advent of the femtosecond (fs) laser and its application to material ablation tasks has proven to be a game changer for materials research. With their extremely rapid milling rates (orders of magnitude faster than traditional ion beam approaches) and minimal heat affected zone (HAZ), the fs-laser has brought about a renaissance in advanced materials characterization capabilities. Recently, a fs-laser mill has been integrated into a commercial focused ion beam and scanning electron microscope (FIB-SEM) instrument, enabling numerous new capabilities, including access to deeply buried structures for advanced analytics such as EDS or EBSD, as well as production of extremely large trenches, cross sections, pillars, and TEM H-bars, all while preserving microstructure and avoiding or reducing FIB polishing. In addition to workflows utilizing the FIB-SEM instrument, this capability enables workflows that span between multiple instruments and the instrument can act as a rapid, targeted sample preparation station to enable a wide range of such workflows. For example, workflows moving from 3D imaging with x-ray microscopy (XRM) into high resolution 2D or 3D imaging with the FIB-SEM can now target deeply buried (more than 50-100 $\mu \mathrm{m}$ below the surface) structures for high resolution analysis via FIB-SEM. Also, workflows moving from micro-scale 3D XRM (samples generally a few millimetres in size) into nano-scale 3D XRM (samples generally $\sim 100 \mu \mathrm{m}$ in size) can leverage the instrument to rapidly prepare nanoscale XRM samples in a targeted manner. Critically for nuclear energy applications, the laser mill is located in a secondary chamber of the FIB-SEM instrument, keeping (potentially radioactive) milling debris from accumulating in the main instrument chamber and prolonging the operational lifetime of the microscope.

This capability dramatically impacts existing materials characterization workflows for nuclear materials research and opens the door to new workflows that were previously impossible or impractical. We use several examples to illustrate these concepts. We first demonstrate the ability to prepare and image massive cross-sections for advanced nuclear fuels such as TRISO particles and structural materials such as steel and aluminum, and show how analytics such as EDS and EBSD can be performed on the native laser-cut surface. Next, we demonstrate the ability to leverage micro-scale x-ray tomography information to target specific, buried regions of interest for preparation and analysis via other techniques like FIBSEM tomography, TEM, or nanoscale x-ray tomography. As a particular example, we demonstrate how this capability can be used to reveal multiscale, correlative porosity information in nuclear grade graphite 
with the intention of building a comprehensive, multiscale model of the pore network character in this material.
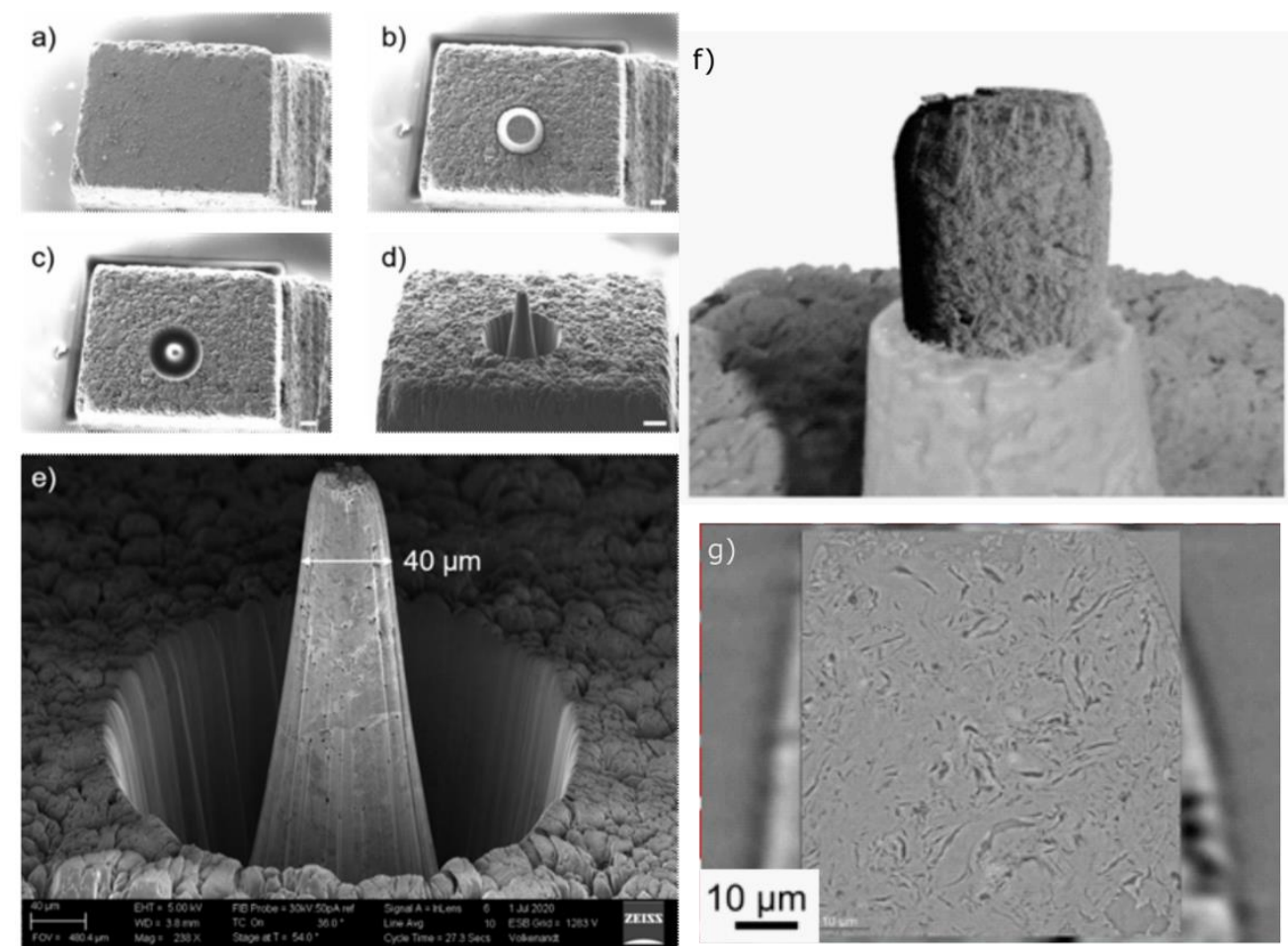

Figure 1. Preparation and analysis of nuclear graphite pillar using fs-laser ablation integrated in a FIB-SEM instrument. Scale bars in a)-d) are $100 \mu \mathrm{m}$.

\section{References}

Tordoff, Ben, et al. "The LaserFIB: new application opportunities combining a high-performance FIB-SEM with femtosecond laser processing in an integrated second chamber." Applied Microscopy 50.1 (2020): 1-11. 